\title{
The association between preconception fruit and vegetable intake in relation to in-vitro fertilization treatment outcome among women attempting fertility
}

\author{
Dimitrios Karayiannis ${ }^{1}$, Meropi Kontogianni ${ }^{1}$, Minas Mastrominas ${ }^{2}$ and Nikos Yiannakouris ${ }^{1}$ \\ ${ }^{1}$ Department of Nutrition and Dietetics, School of Health Sciences and Education, Harokopio University, Athens, \\ Greece and \\ ${ }^{2}$ Embryogenesis Assisted Conception Unit., Athens, Greece
}

\begin{abstract}
Introduction: Adequate intake of fruits and vegetables $(\mathrm{F} \& \mathrm{~V})$ has been long promoted as a public health priority based on the evidence supporting various health benefits attributed to these food groups. Their effects on reproductive outcomes, however, remain poorly explored. F\&V intake has been positively associated with embryo quality in women undergoing in-vitro fertilization (IVF) but there is lack of data regarding potential associations with IVF final outcomes (clinical pregnancy and live birth). Thus, the aim of this study was to evaluate habitual F\&V intake in relation to clinical pregnancy and live birth among women undergoing infertility treatment.
\end{abstract}

Materials and Methods: Nulliparous non-obese women $\left[\mathrm{n}=244,22-41\right.$ years old, body mass index $\left.(\mathrm{BMI})<30 \mathrm{~kg} / \mathrm{m}^{2}\right] \mathrm{undergoing}$ their first IVF cycle were recruited from an Assisted Conception Unit in Athens, Greece, between November 2013 and September 2016. Habitual F\&V intake was estimated via a validated for the Greek population semi-quantitative food-frequency questionnaire and expressed in servings/day. Intermediate outcomes (i.e. oocyte yield and embryo quality measures) and clinical endpoints (implantation, clinical pregnancy and live birth) were prospectively recorded. Generalized linear models adjusting for age, ovarian stimulation protocol, BMI, physical activity, anxiety levels, infertility diagnosis, caloric intake and supplements use were used to test associations between F\&V intake and IVF outcomes.

Results: Overall, 229 women (93.9\%) had an embryo transfer, $104(42.6 \%)$ achieved a clinical pregnancy and 99 (40.5\%) had a live birth. Women with a clinical pregnancy and live birth did not differ in age, BMI, smoking habits, physical activity or stress levels, compared to those who did not achieve pregnancy (all $\mathrm{P}>0.05$ ). Compared to women with IVF failure, those achieving clinical pregnancy and live birth reported consuming more fruits (median values: 1.77 vs. 1.49 servings/day and 1.77 vs. 1.41 servings/day, respectively) and more vegetables (1.79 vs. 1.37 servings/day and 1.89 vs. 1.36 servings/day, respectively), all $\mathrm{P}<0.05$. No significant association between F\&V intake and IVF intermediate outcomes was recorded. The multivariable-adjusted relative risk $(95 \%$ confidence interval) for clinical pregnancy for increasing intake of fruit and vegetable (servings/day, continuous) was 1.23 (1.06-1.44) and 1.25 (1.02-1.57), respectively, and for live birth it was $1.29(1.10-1.51)$ and $1.36(1.10-1.71)$, respectively.

Discussion: Higher pre-treatment F\&V was related to higher probability of clinical pregnancy and live birth among women undergoing IVF. These results highlight the importance of dietary influences on fertility and suggest the need for additional research on the effects of $F \& V$ intake on reproductive endpoints in women conceiving through infertility treatments.

\section{Conflict of Interest}

There is no conflict of interest 